\title{
Robust Position and Velocity Estimation Methods in Integrated Navigation Systems for Inland Water Applications
}

D. Arias-Medina, M. Romanovas, I. Herrera-Pinzón, R. Ziebold

German Aerospace Centre (DLR)

IEEE/ION PLANS 2016

Integrated Inertial Navigation

13. April 2016

DLR 


\section{Agenda}

$\diamond$ Introduction

- Motivation

- Objectives

$\diamond$ Methods

- Robust Estimation

- Sensor Fusion

$\diamond$ Tests and Results

$\diamond$ Summary and Outlook

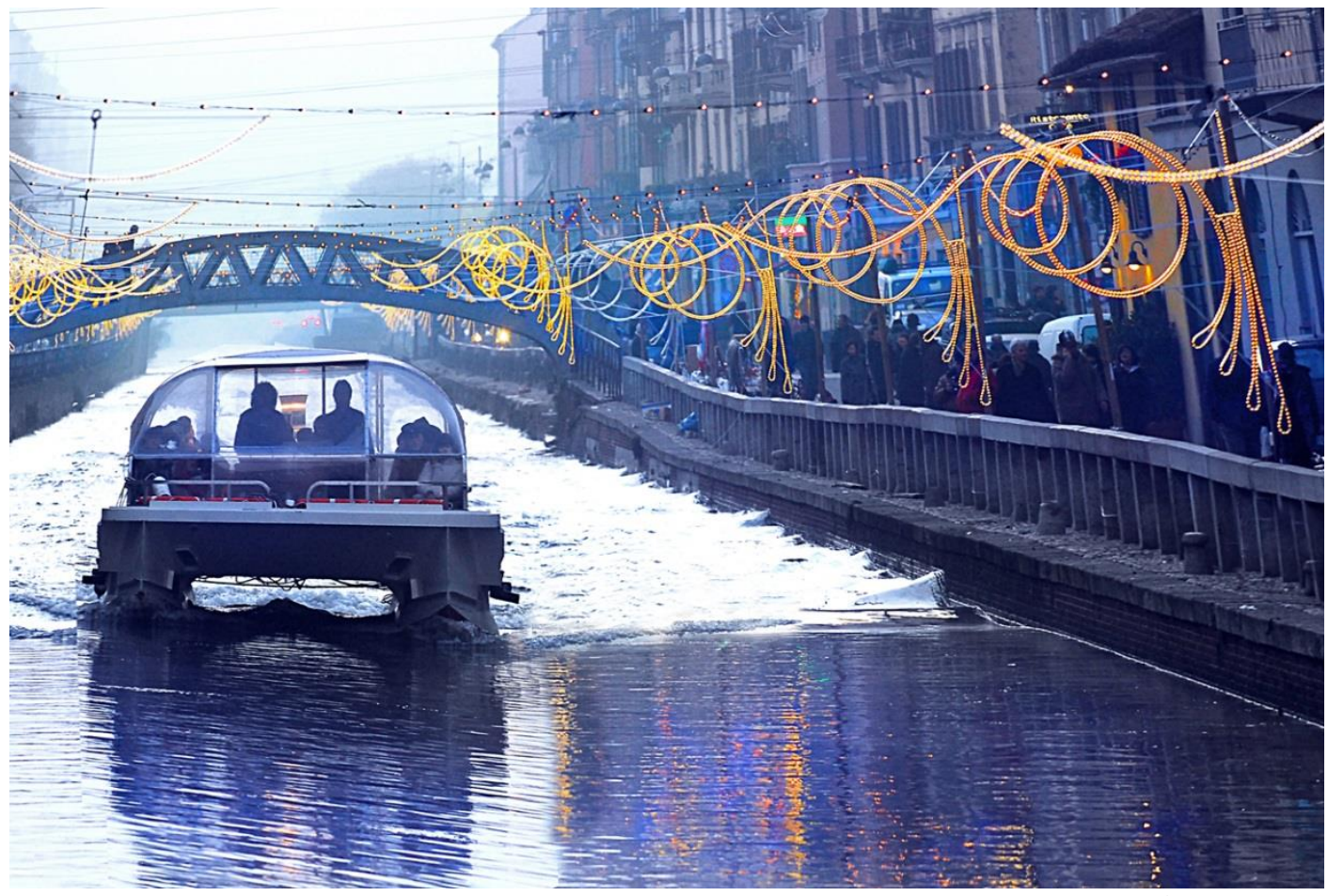

source: www.waterways-forward.eu 


\section{Agenda}

$\diamond$ Introduction

- Motivation

- Objectives

$\diamond$ Methods

- Robust Estimation

- Sensor Fusion

$\diamond$ Tests and Results

$\diamond$ Summary and Outlook

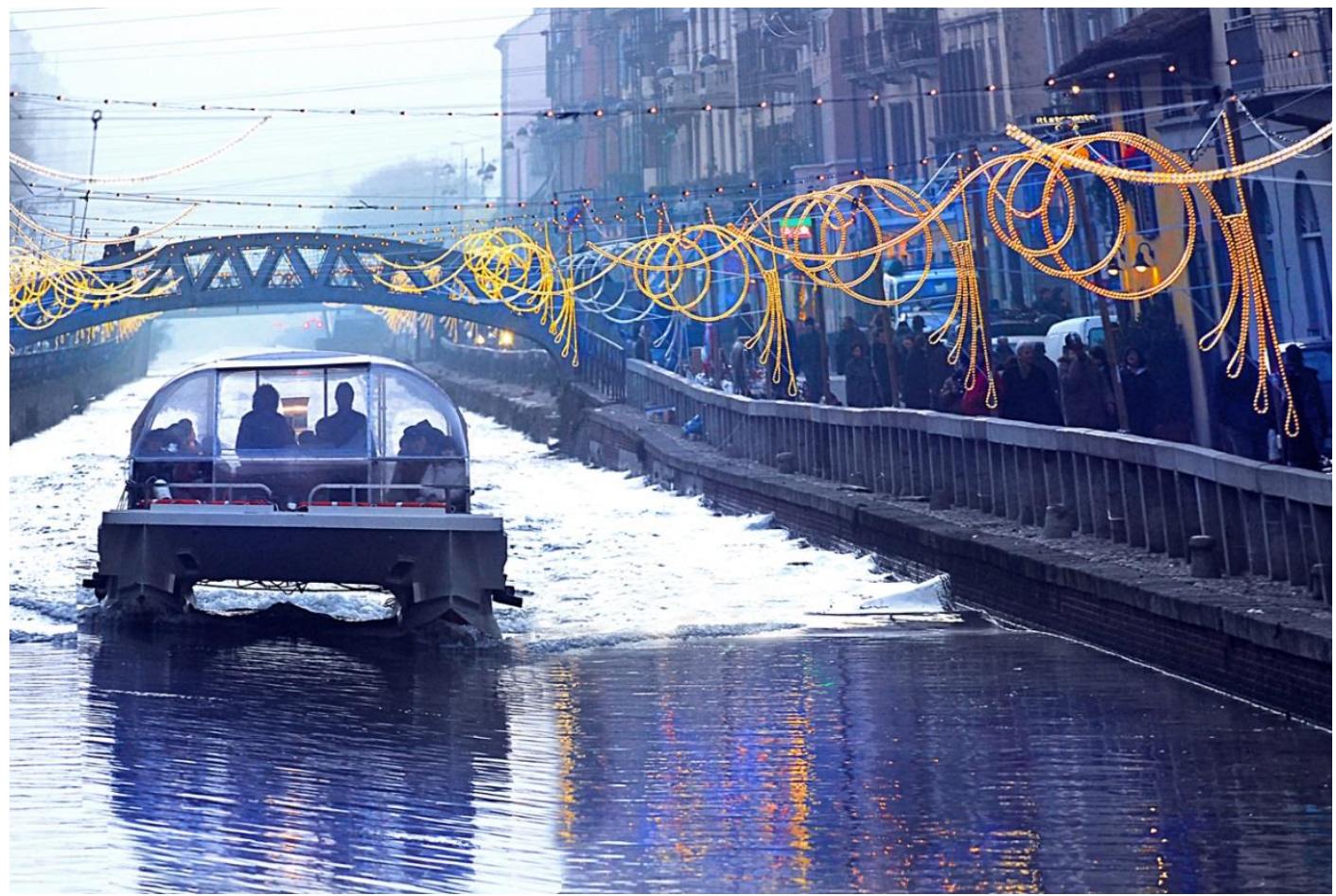

source: www.waterways-forward.eu 


\section{Motivation}

- Maritime transport is the backbone of international trade and the global economy:

- $\sim 80 \%$ global trade by volume is made by sea

- Around 400 Mio. passengers move through European ports each year

Nautical Transport Systems are essential for the global economic development, competitiveness and prosperity

\section{Unfortunately...}

- The number of shipping accidents is not decaying over the years 


\section{Motivation}
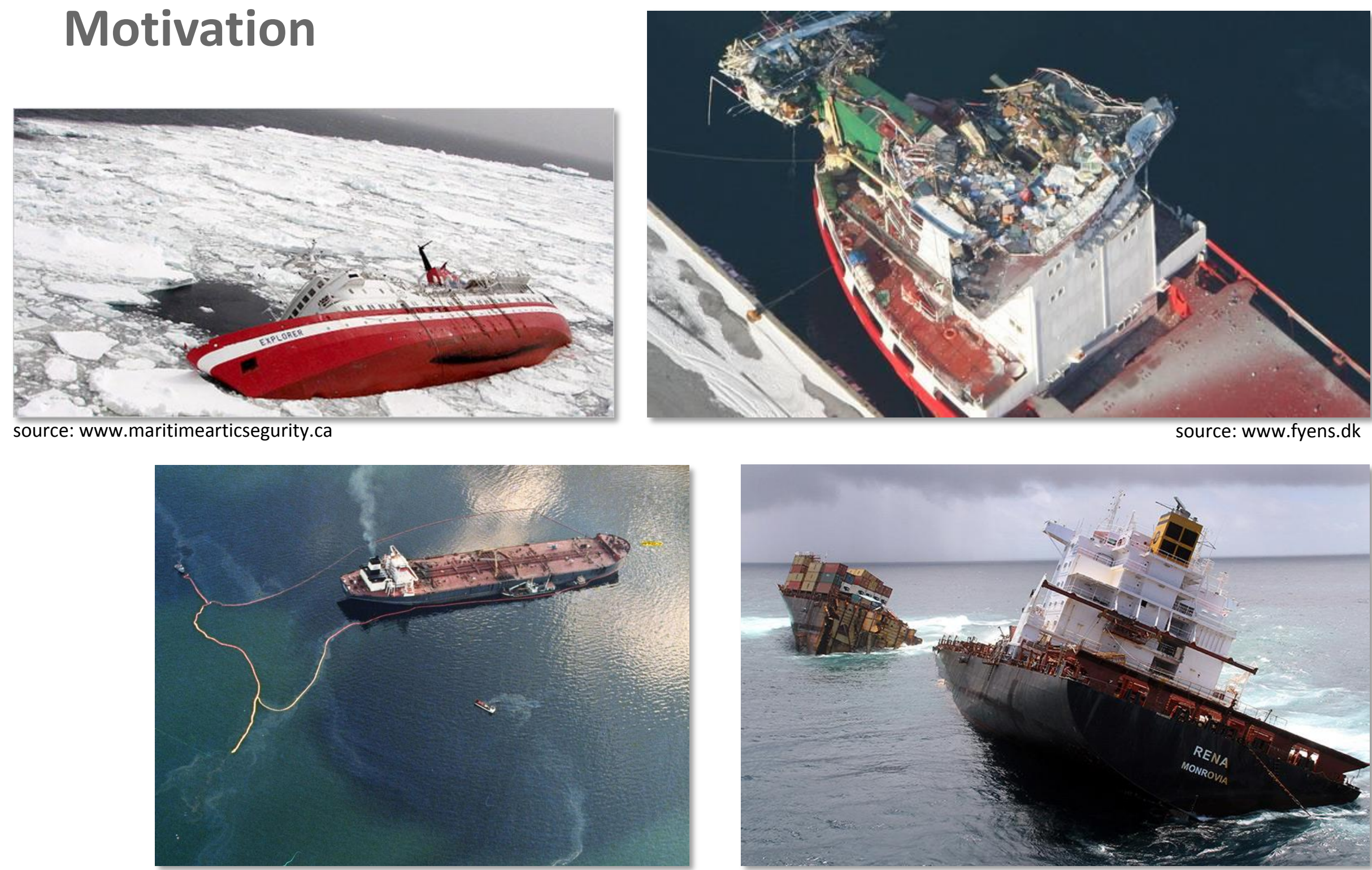

source: www.abc.es

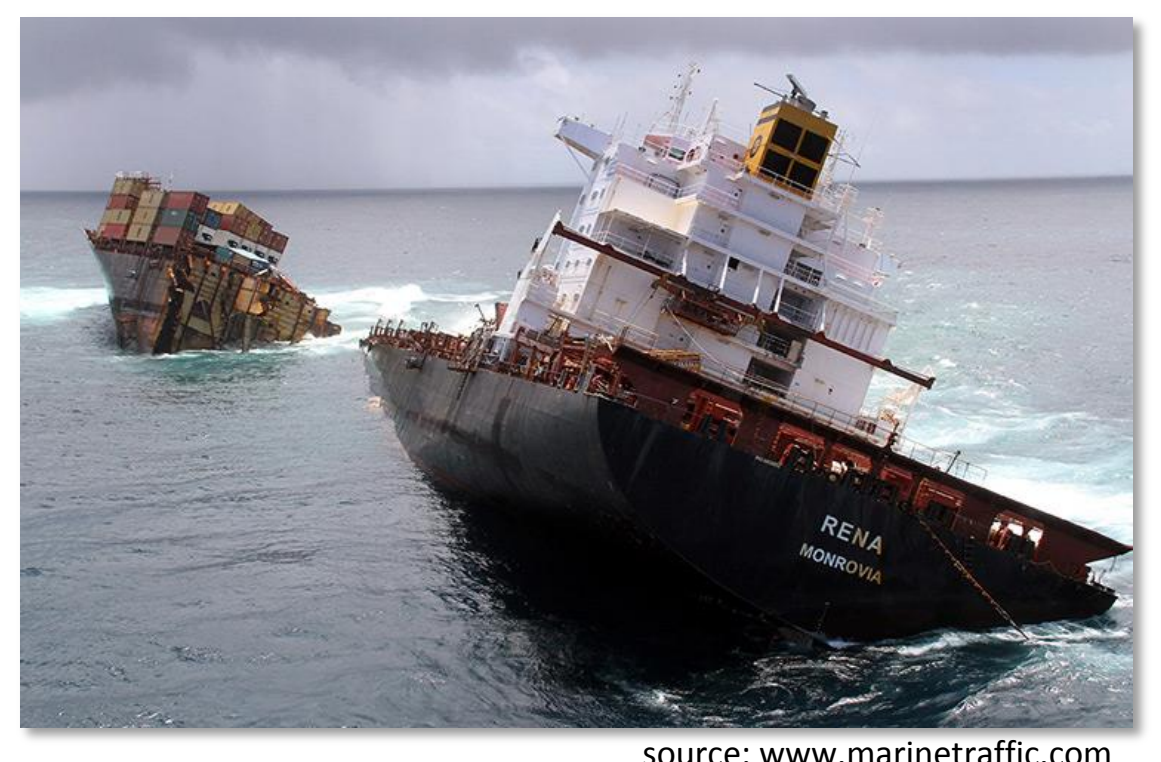

source: www.marinetraffic.com

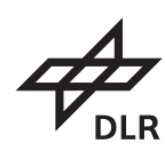




\section{Motivation}

- Kiel Canal: world busiest artificial waterway

- Collision of two medium-sized vessels at night

- Positioning systems on both vessels showed a safe passing-distance

- RADAR was not used

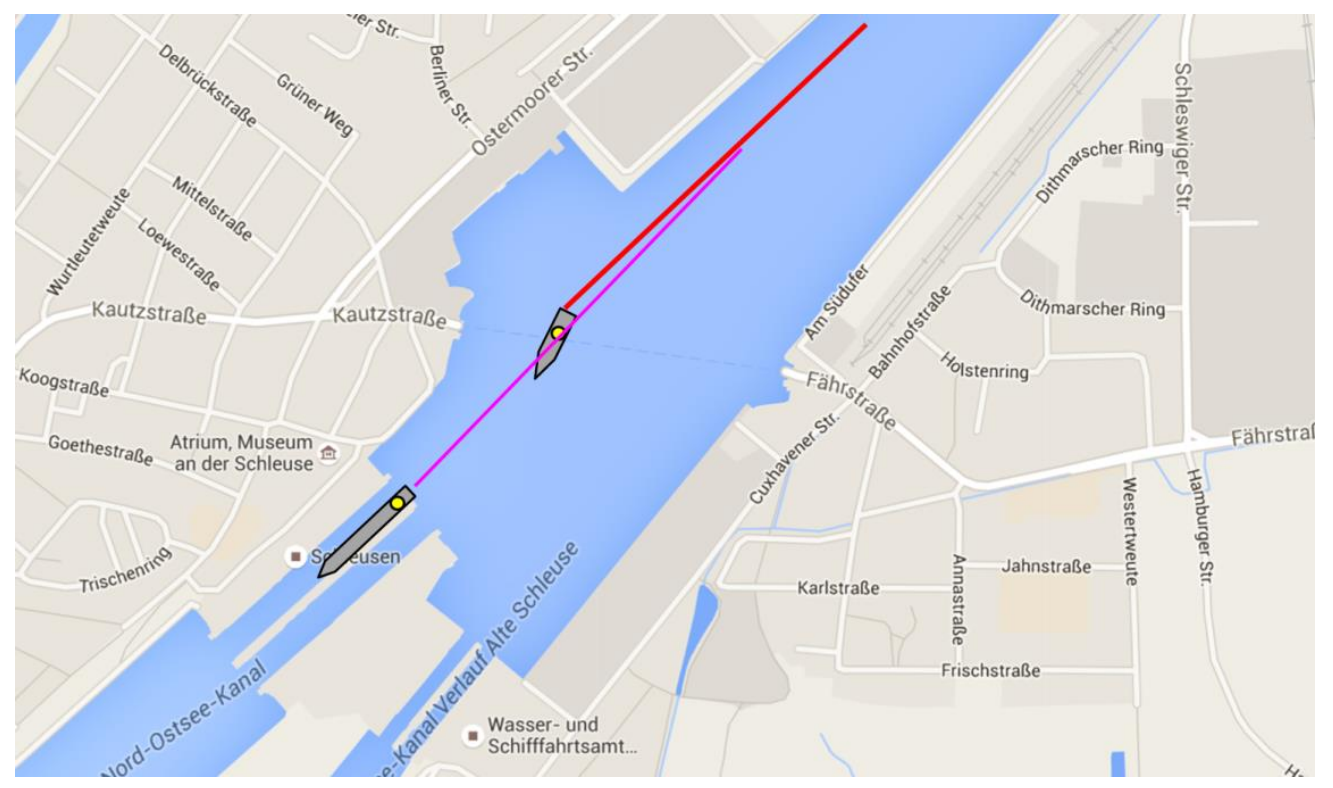

Global Navigation Satellite Systems (GNSS) are the cornerstone and main information supplier for Positioning, Navigation and Timing (PNT) in maritime systems. 


\section{Motivation}

- The performance of satellite - based navigation can be easily disturbed due to space weather events, jamming, reflection of the signals, ...

- Classical positioning is solved applying a Least Squares (LS) method $\rightarrow$ single contaminated signal induce large errors in the position

- Receiver Autonomous Integrity Monitoring (RAIM) is the standard for GNSS fault detection but... it cannot handle multiple simultaneous faults!

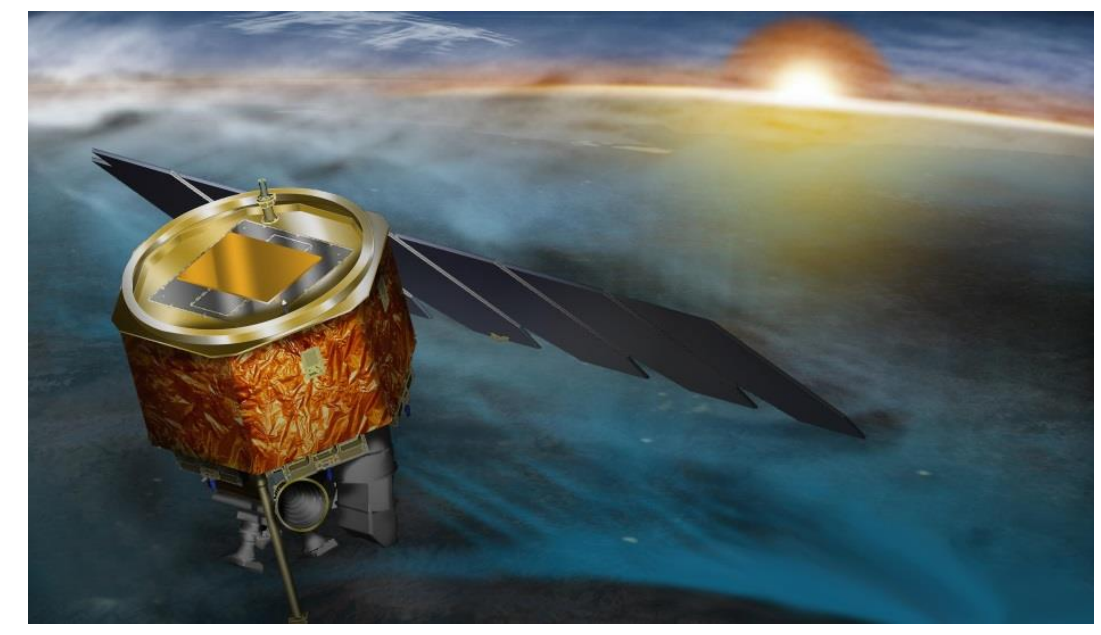

- Satellite - based navigation lacks robustness: capability of a system to continue operating despite abnormalities

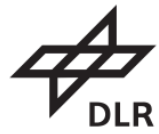




\section{Objectives}

- What do we want?

Provide a reliable navigation solution mitigating GNSS faulty signals

-What is the problem?

* Multiple simultaneous faulty signals, specially in urban canyons or waterways

× Standard RAIM is not sufficient

- What is our solution?

- Implementation of robust estimators for the positioning problem

- Integration of these algorithms within an inertial + satellite based navigation 


\section{Agenda}

$\diamond$ Introduction

- Motivation

- Objectives

$\diamond$ Methods

- Robust Estimation

- Sensor Fusion

$\diamond$ Tests and Results

$\diamond$ Summary and Outlook

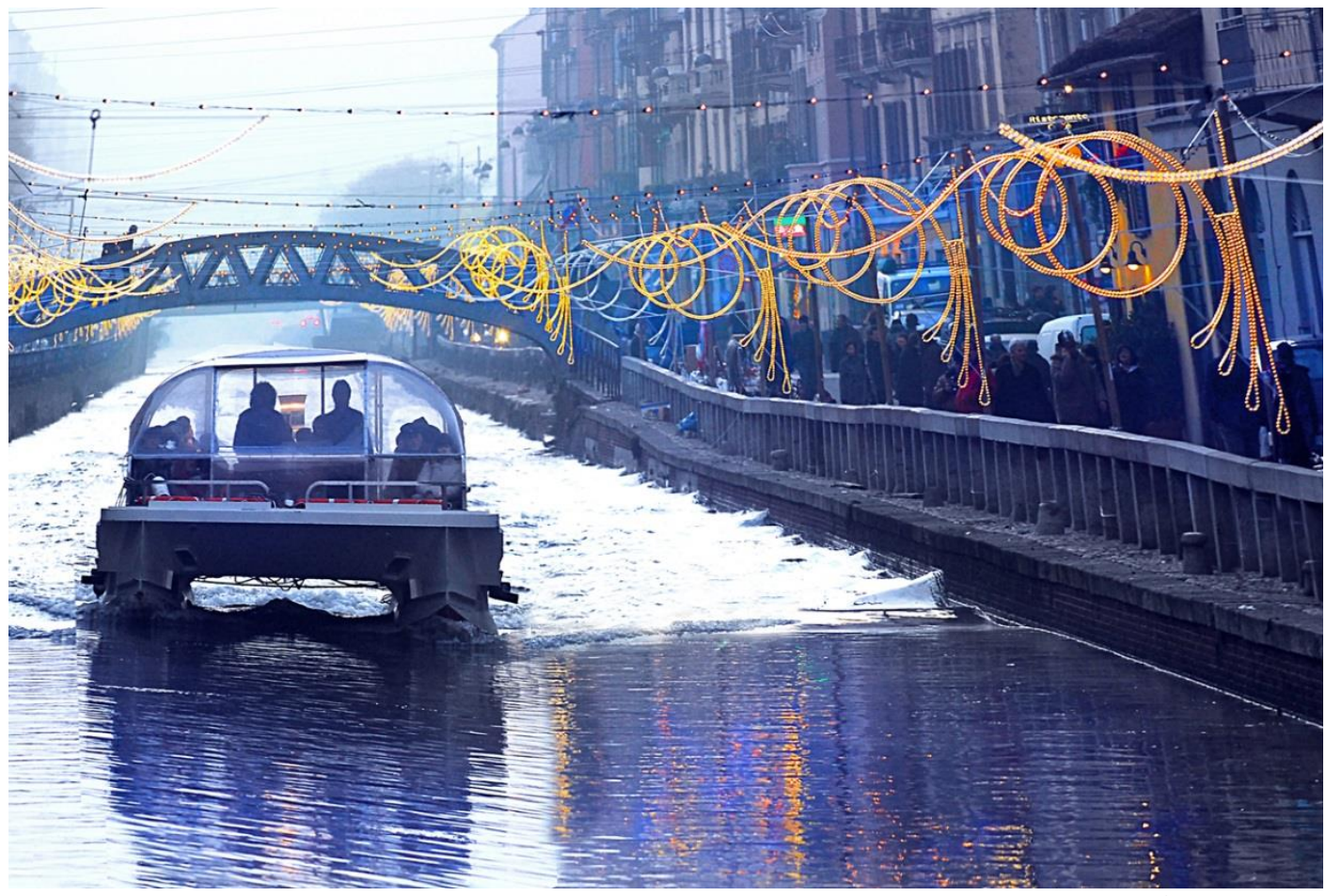




\section{Robust Estimation}

- GNSS positioning problems are generally solved $\rightarrow$ LS estimator

- In a LS, it is assumed that the noises are Gaussian...

But this is often not the case!

\section{Clue definitions}

- Outliers - observations that appear unusually large or small and "out of place"

- Breakdown Point $\epsilon^{*}$ - smallest percentage of contaminated data that can cause the estimator to take arbitrarily large values

- Gaussian Efficiency - similarity of a method to classical LS under Gaussian conditions
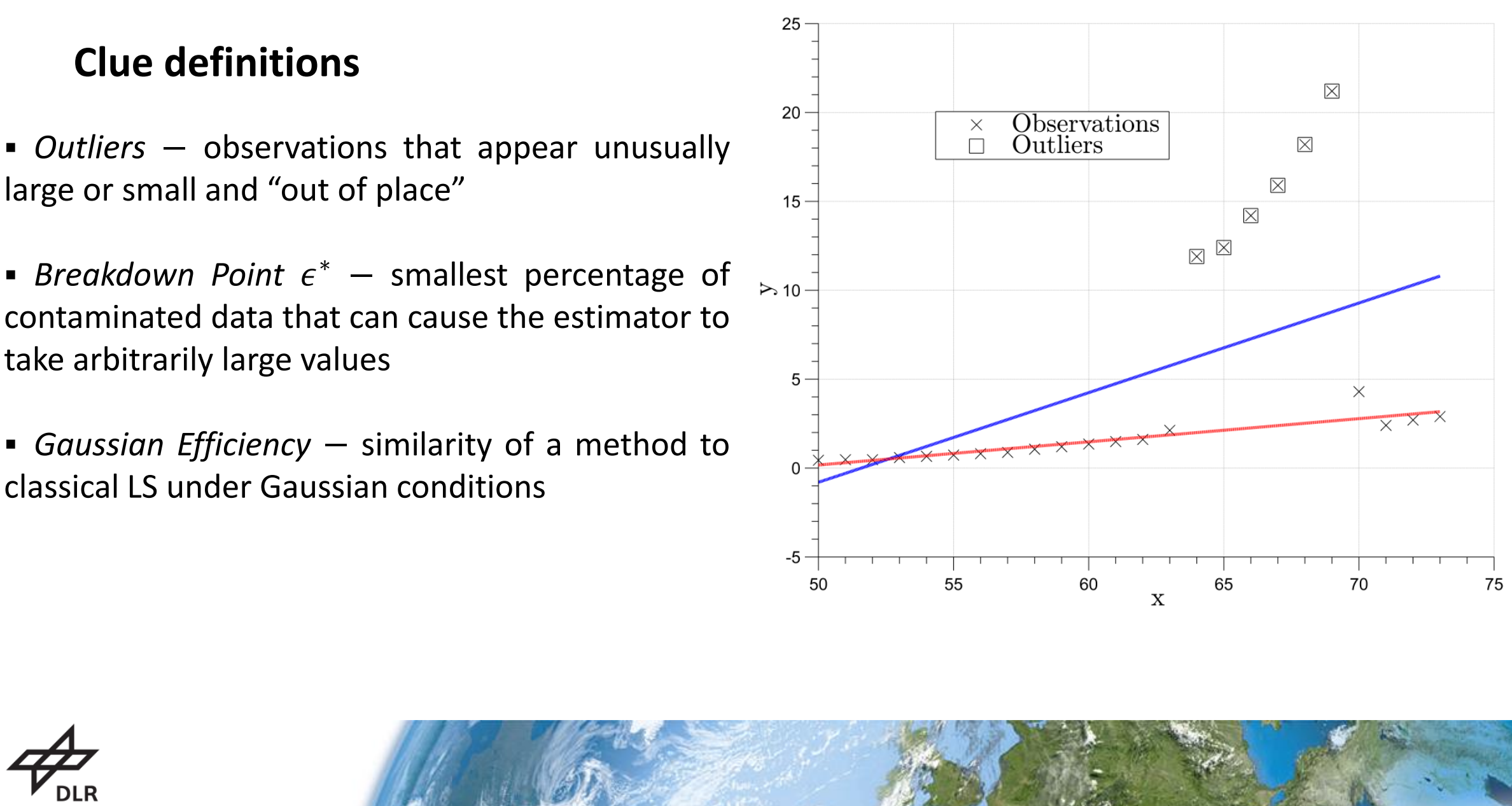


\section{Robust Estimation}

- Overpassing the limitations of LS for regression has concerned mathematicians and engineers for years...

\section{Iteratively Reweighted Least Squares (IRLS)}

- Full set Approactimator observations are used to compute sol(ntipn, observations with large residu trif $_{1}$ are $\hat{o}_{\mathrm{d}}$ 'ownweighted

- Appealicoimplesmintatipn for its similarity to regular LS $n$

- Gaussian éfficient

$$
\min \sum_{\text {efficient }}^{n} w\left(x_{i}\right) \rho\left(\frac{r_{i}}{w\left(x_{i}\right) \hat{\sigma}}\right), \quad \epsilon^{*}=\frac{1}{n+1}
$$

- Breakdown point $\epsilon$ not very high

$$
\operatorname{mins}\left(r_{1}, \ldots, r_{n}\right), \quad \epsilon^{*}=\left(\frac{1}{2}-p+2\right) / n
$$

\section{Best Subset Selection}

- Bottom - up approach $\rightarrow$ from $n$ observations, $\left(\begin{array}{l}n \\ p\end{array}\right)$ subsets are made $>$ Least Median of Squares (LMS)

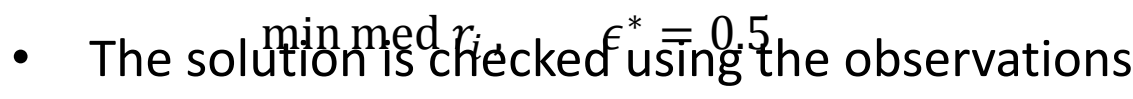
not taking part in the solution $>$ Least Trimmed of Squares (LTS)

- The best subset is the one to

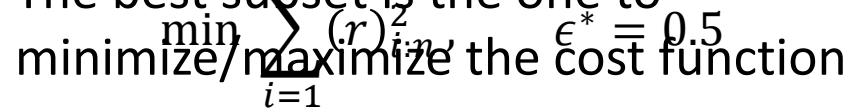

- Breakdown point $\epsilon^{*}$ up to $50 \%$

- Low Gaussian efficiency

There are also other approaches...

- Receiver Autonomous Integrity Monitoring (RAIM)

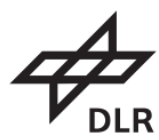




\section{Kalman Filtering for Sensor Fusion}

- Standard approach for multi-sensor fusion and navigation

- Incorporate of all the available information (uncertainties, noise statistics, dynamical models, kinematic constraints) in a statistically consistent way

- Kalman Filter (KF) is valid for linear problems $\rightarrow$ Extended \& Unscented KF (UKF, EKF)
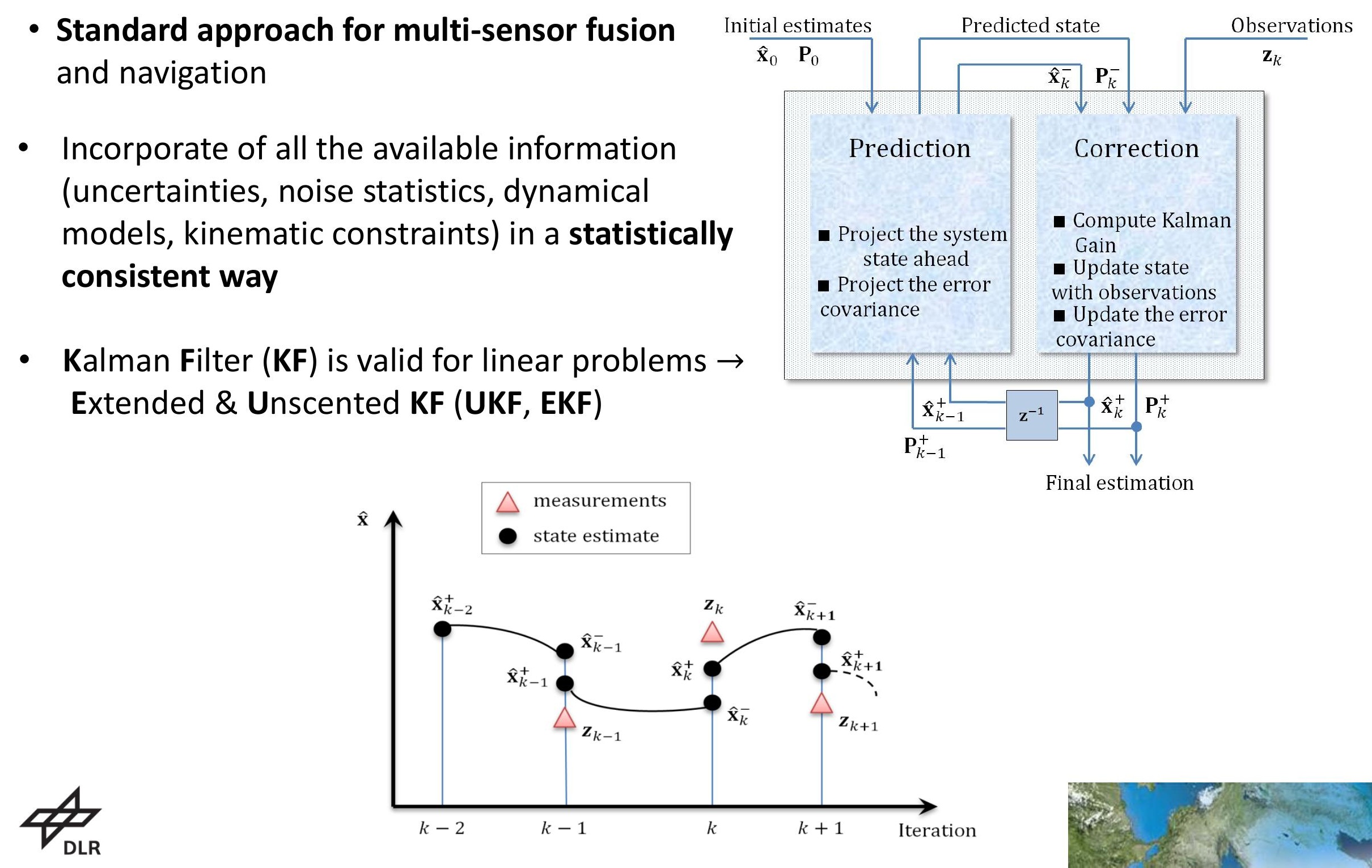

Final estimation 


\section{UKF for IMU/GNSS Navigation}

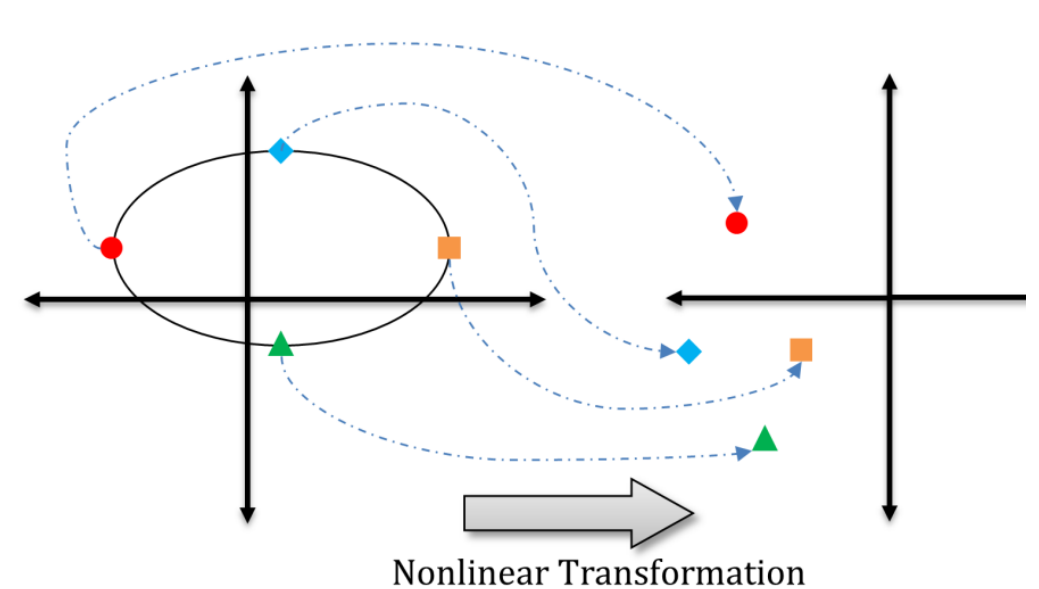

- The state is represented by a set of sigma points $\rightarrow$ propagated through the nonlinear functions

- The mean and covariance of the solution are reconstructed back from the sigma points

- Attention: this is not a Monte Carlo method!

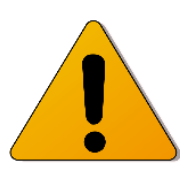

Nonlinear Transformation

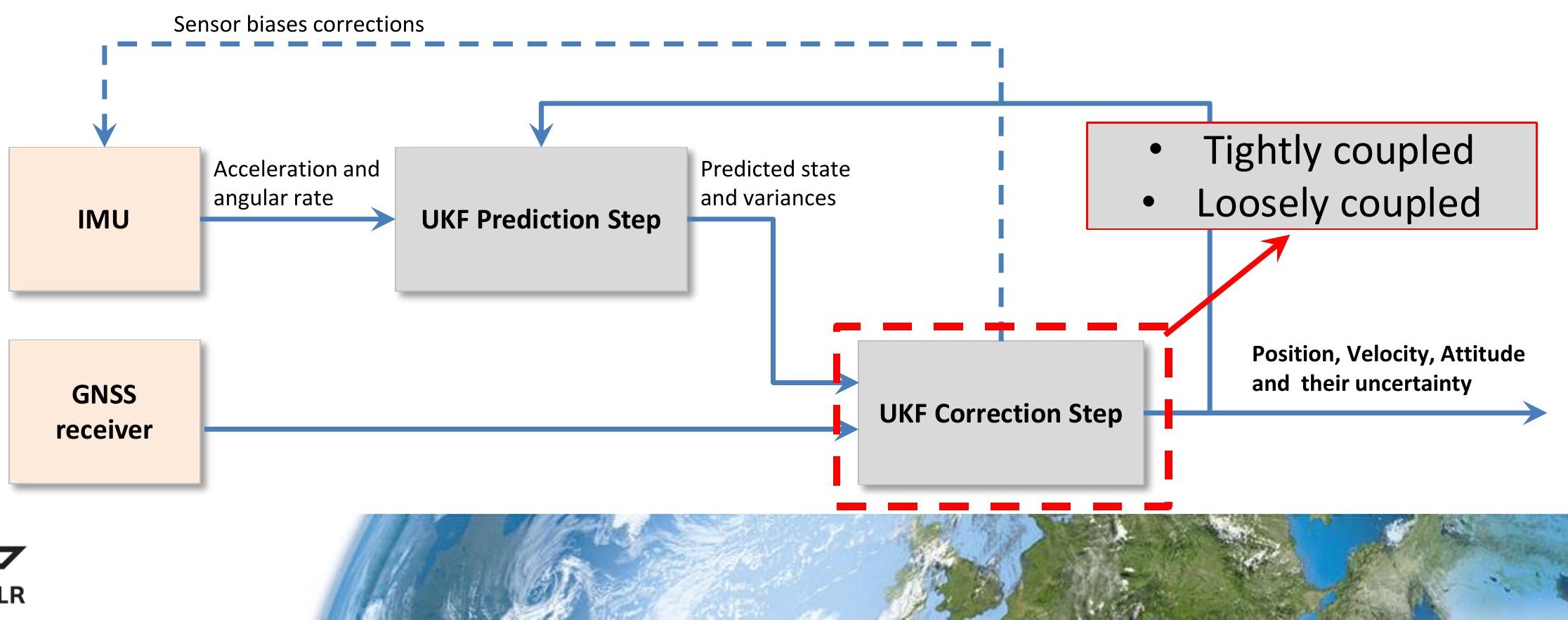




\section{Agenda}

$\diamond$ Introduction

- Motivation

- Objectives

$\diamond$ Methods

- Robust Estimation

- Sensor Fusion

$\diamond$ Tests and Results

$\diamond$ Summary and Outlook

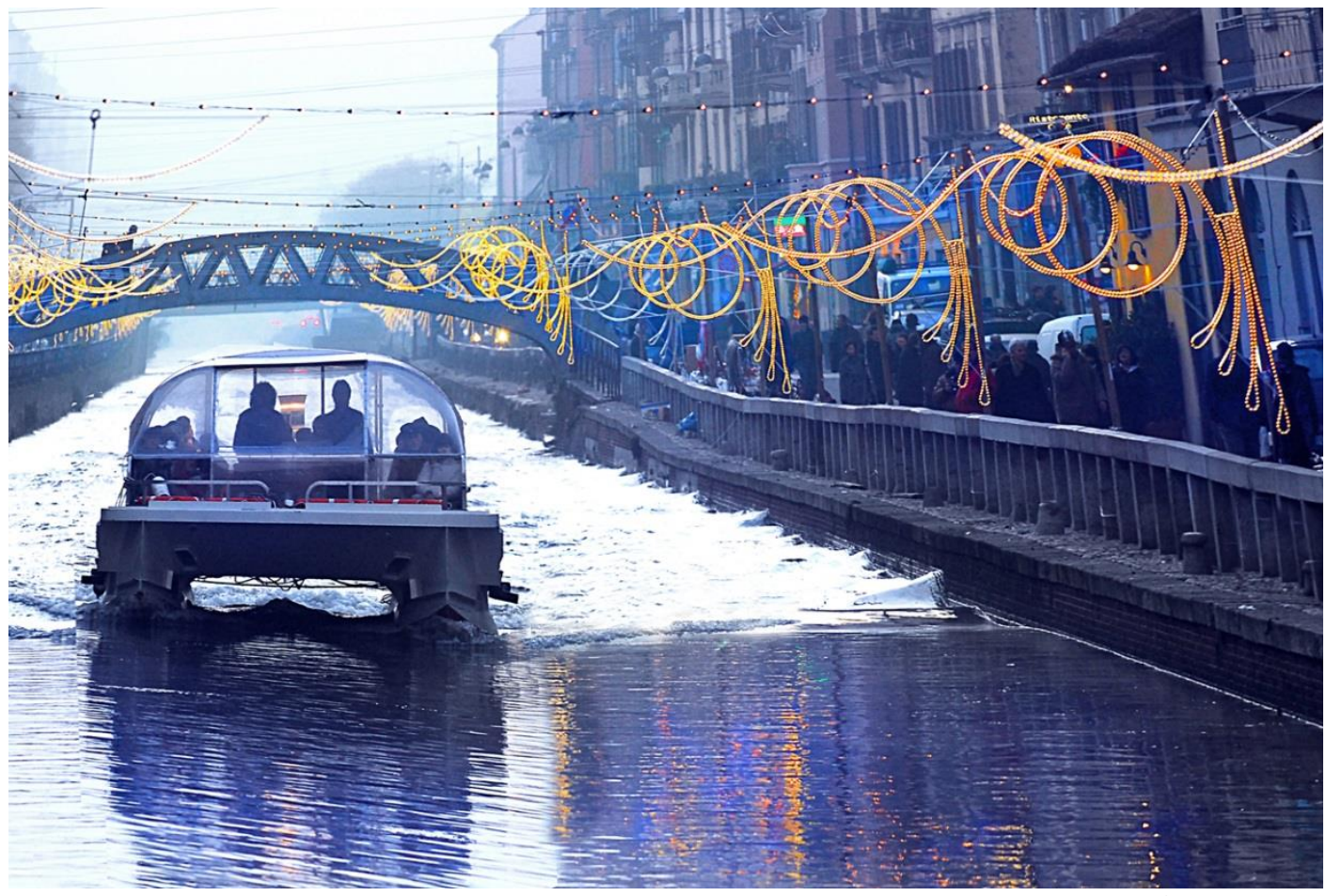




\section{Experiment Setup}

- The test scenario is the Moselle River in Koblenz (Germany)

- Vessel "MS BINGEN" performed 8 - shaped trajectory passing under the bridges Equipment of vessel:

- $3 x-$ GNSS antennas, update rate $1 \mathrm{~Hz}$

- $1 x$-inertial sensors: gyroscope and accelerometer, update rate $200 \mathrm{~Hz}$
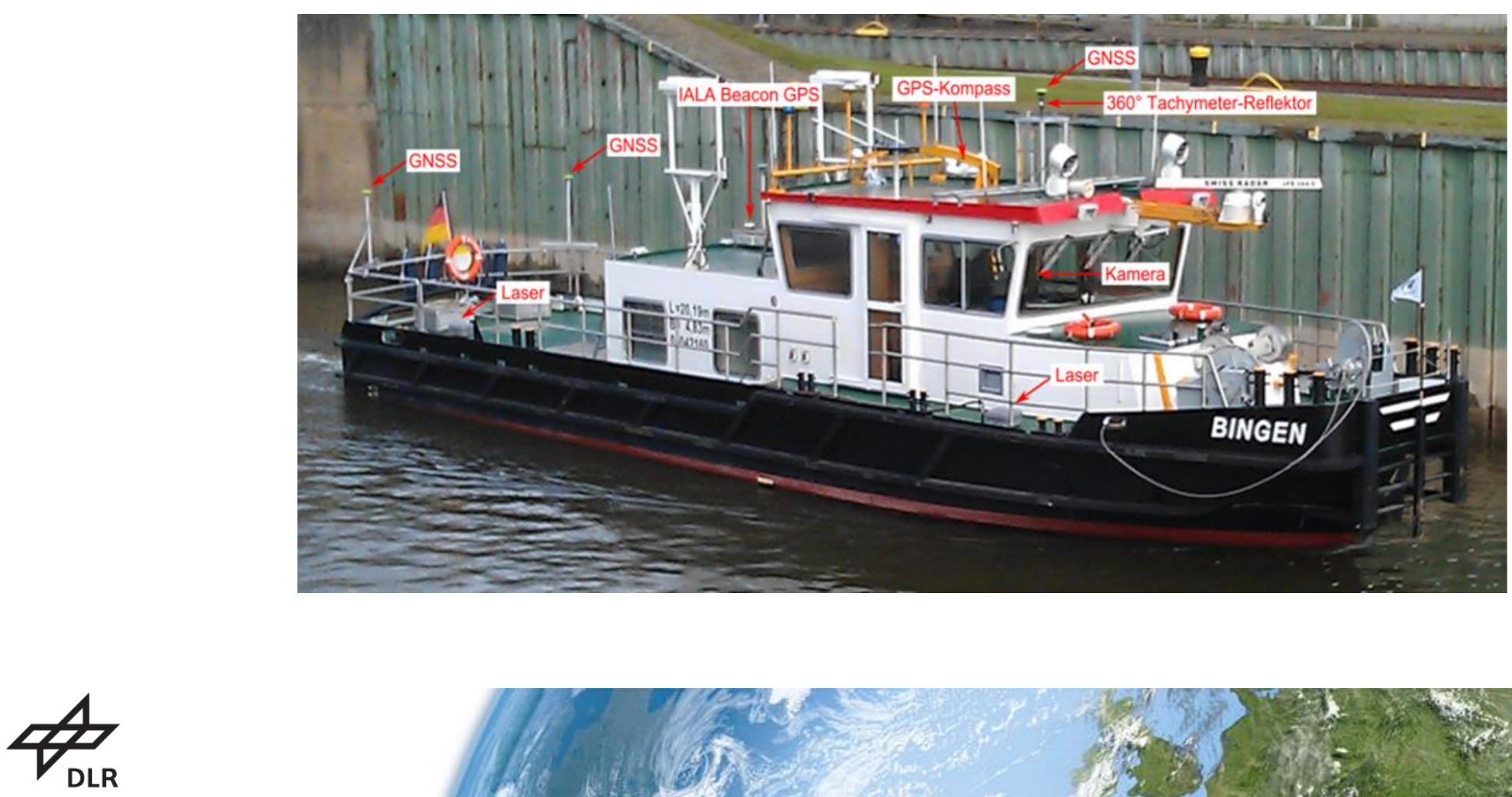


\section{Moselle River Scenario}

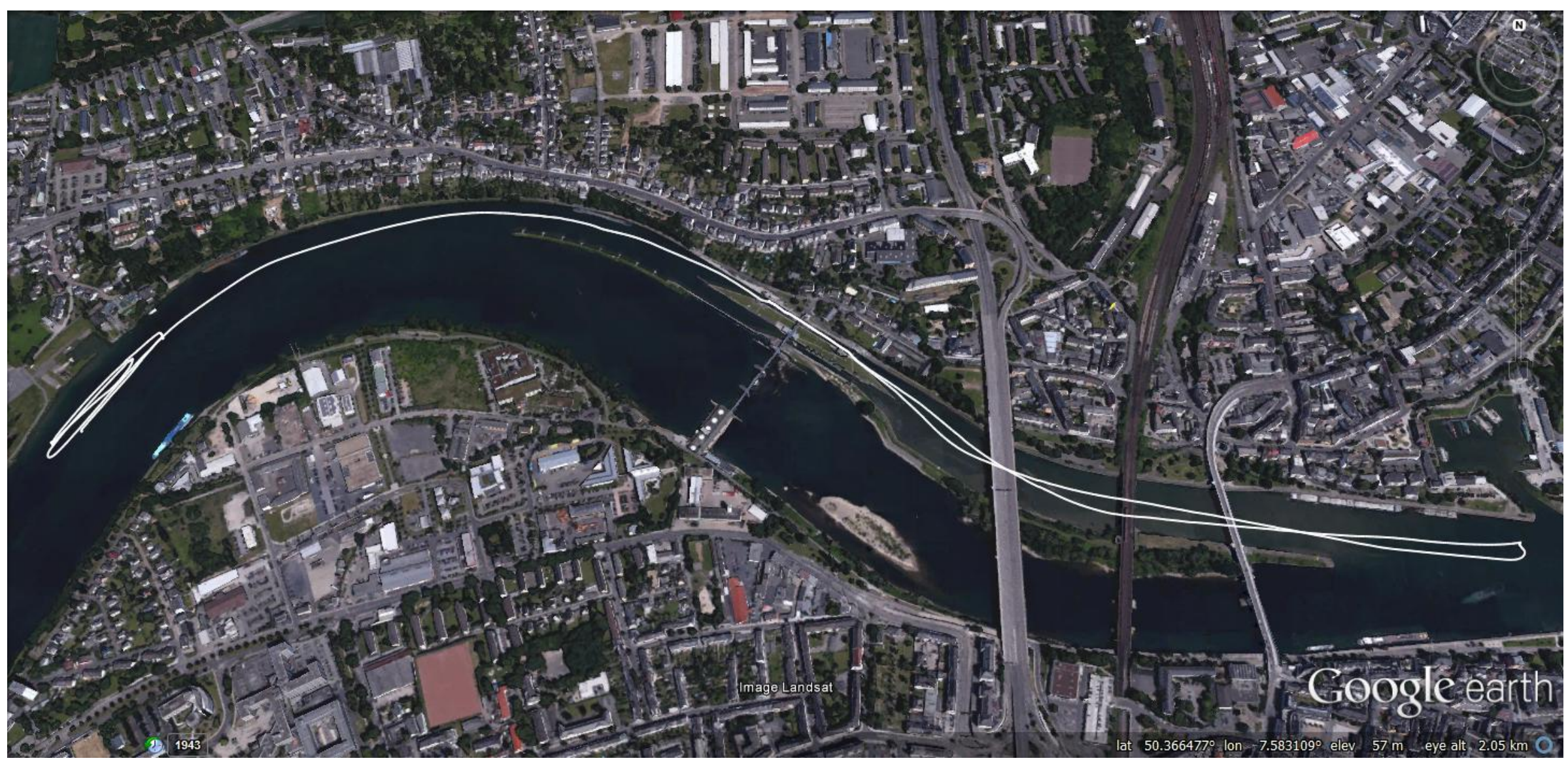

$f_{\mathrm{DLR}}$ 


\section{Robust Method Comparison}

$$
\begin{array}{ll}
\bigcap_{\text {LS }} & \bigcap^{\text {s }- \text { est }} \\
\bigcap_{\text {LMS }} & \bigcap_{\text {RAIM }}
\end{array}
$$
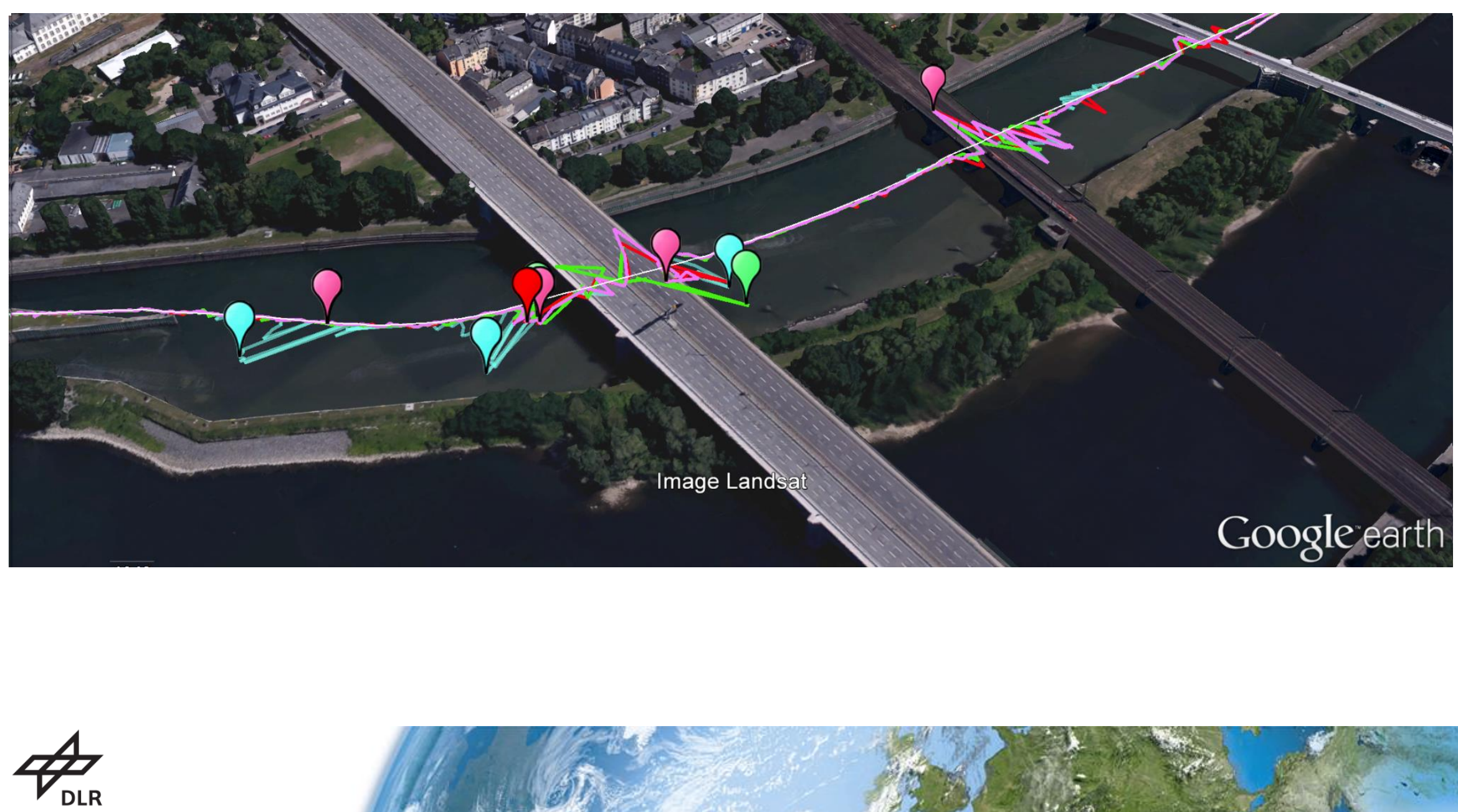


\section{Discussion on Robust Estimation}

\section{Statistics on the Robust Methods performance}

\begin{tabular}{c||ccc}
\hline \multirow{2}{*}{ Method } & Mean [m] & RMS [m] & Max [m] \\
& & & \\
\hline \hline & & & \\
SPP & 2.9 & 3.5 & 50.7 \\
S & 2.4 & 3.4 & 34.0 \\
LMS & 2.4 & 3.0 & 45.4 \\
RAIM & 2.3 & & \\
\hline
\end{tabular}

$\checkmark$ Robust techniques perform better than regular Single Point Positioning (SPP)

$\checkmark$ The mean error is reduced and the maximum error is $15 \mathrm{~m}$ smaller

- LMS and S estimator have a similar performance but...

- LMS requires higher computation

- LMS has a low Gaussian efficiency 


\section{UKF Performance}

- Comparison of the different UKF designs:

\section{$>$ Tightly Coupled UKF}

State for the Tightly Coupled Architecture UKF

\begin{tabular}{|c|c|c|c|c|}
\hline State & Covariance & Variable & Symbol & Coordinate System \\
\hline $1: 4$ & $1: 3$ & Attitude Quaternion & $q$ & From B-frame to ECEF \\
$5: 7$ & $4: 6$ & Velocity & $v$ & ECEF \\
$8: 10$ & $7: 9$ & Position & $p$ & ECEF \\
$11: 13$ & $10: 12$ & Gyroscope Offset & $b_{\omega}$ & B-frame \\
$14: 16$ & $13: 15$ & Accelerometer Offset & $b_{a}$ & B-frame \\
17 & 16 & Clock offset & $c \delta t$ & - \\
18 & 17 & Clock rate & $c \dot{\delta} t$ & - \\
\hline
\end{tabular}

\section{Loosely Coupled UKF + a) classical LS b) robust scheme}

State for the Loosely Coupled Architecture UKF

\begin{tabular}{|c|c|c|c|c|}
\hline State & Covariance & Variable & Symbol & Coordinate System \\
\hline $1: 4$ & $1: 3$ & Attitude Quaternion & $q$ & From B-frame to ECEF \\
$5: 7$ & $4: 6$ & Velocity & $v$ & ECEF \\
$8: 10$ & $7: 9$ & Position & $p$ & ECEF \\
$11: 13$ & $10: 12$ & Gyroscope Offset & $b_{\omega}$ & B-frame \\
$14: 16$ & $13: 15$ & Accelerometer Offset & $b_{a}$ & B-frame \\
\hline
\end{tabular}




\section{UKF Performance Discussion}

Statistics on the KF performance

\begin{tabular}{c||ccc}
\hline Method & Mean [m] & RMS [m] & Max [m] \\
& & & \\
\hline \hline & & & \\
SPP & 2.9 & 4.5 & 50.7 \\
TC UKF & 3.0 & 3.8 & 18.3 \\
LC UKF & 3.0 & 3.70 & 17.0 \\
LC-S UKF & 2.3 & 2.6 & 9.1 \\
\hline
\end{tabular}

$\checkmark$ Kalman filtering provides a smooth position solution $\rightarrow$ largest errors are eliminated

$\checkmark$ The inclusion of robust estimator $\rightarrow$ significant improvement in the position error

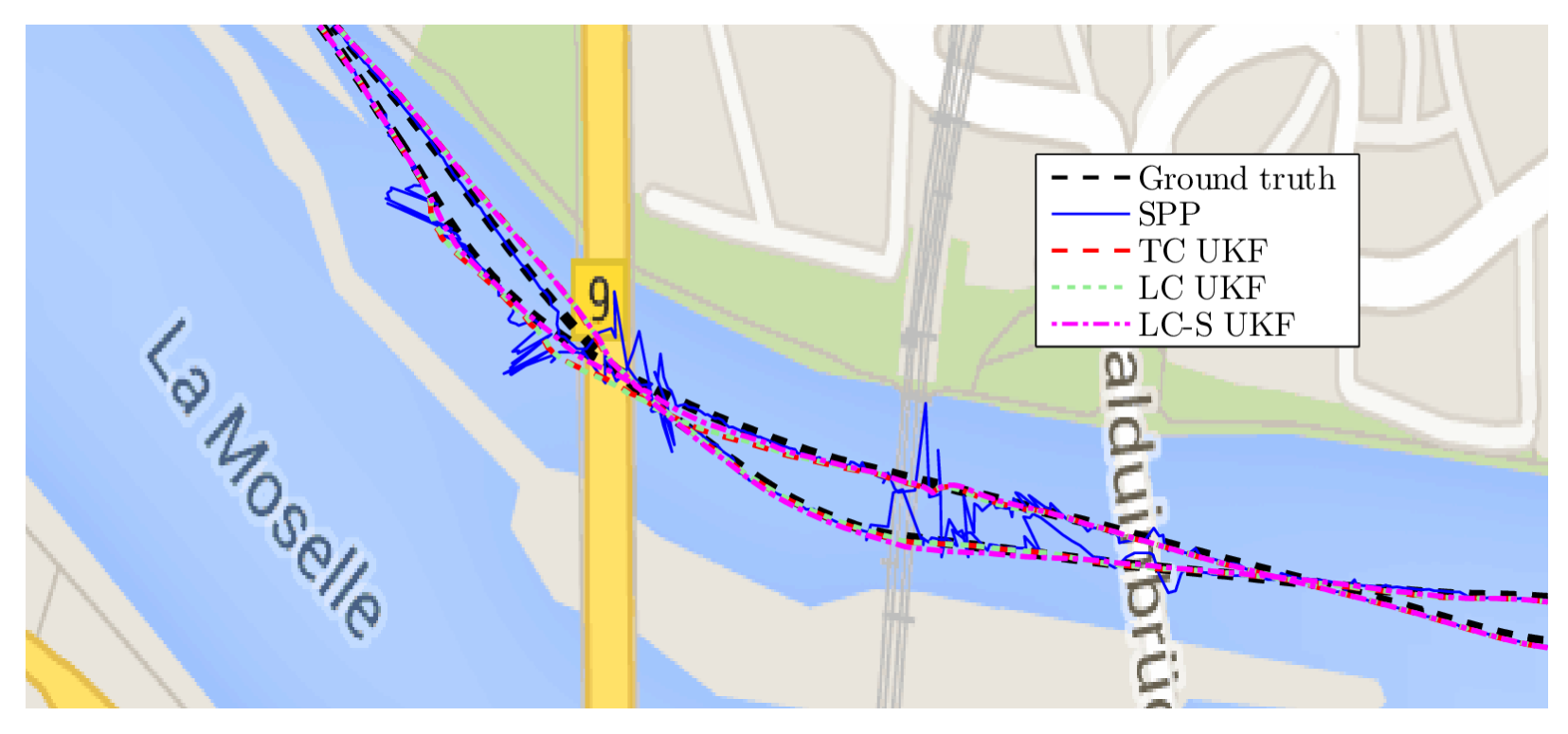




\section{Agenda}

$\diamond$ Introduction

- Motivation

- Objectives

$\diamond$ Methods

- Robust Estimation

- Sensor Fusion

$\diamond$ Tests and Results

$\diamond$ Summary and Outlook

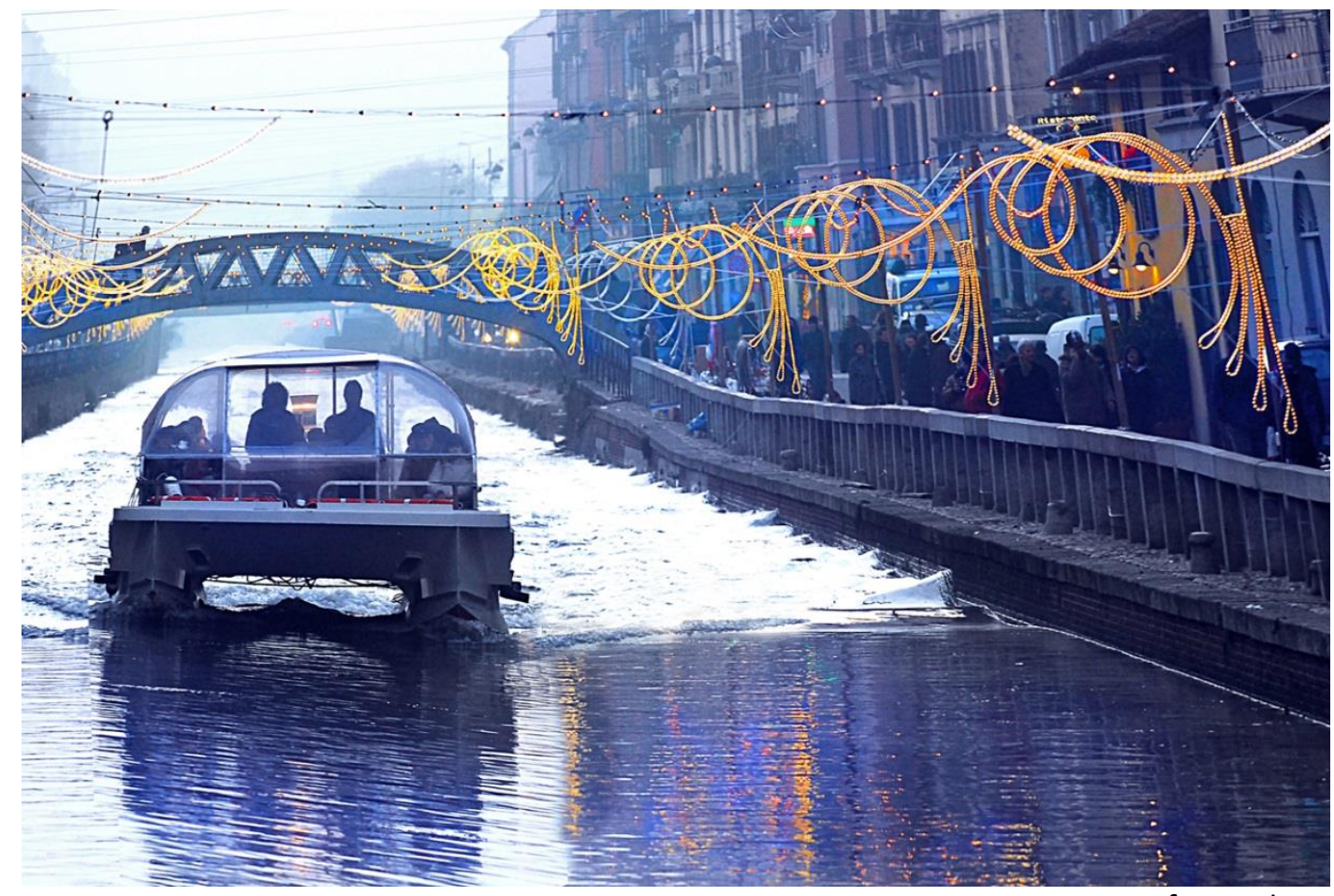




\section{Conclusions}

- Review on the techniques for GNSS fault mitigation

- Integrated navigation fusing IMU+GNSS sensors using UKF

- Evaluation of the algorithms using real data

$\checkmark$ Promising performance improvement vs. classical LS

$\checkmark$ Great benefits of the use of robust schemes + KF 


\section{Future Work}

- Extension to Multi - antenna, Multi - constellation, Multi - frequency (MMM)

- Robust schemes lack any kind of integrity monitoring $\rightarrow$ user gets warned if position estimation is not reliable

- Implementation of the robust estimation in the tightly coupled UKF 

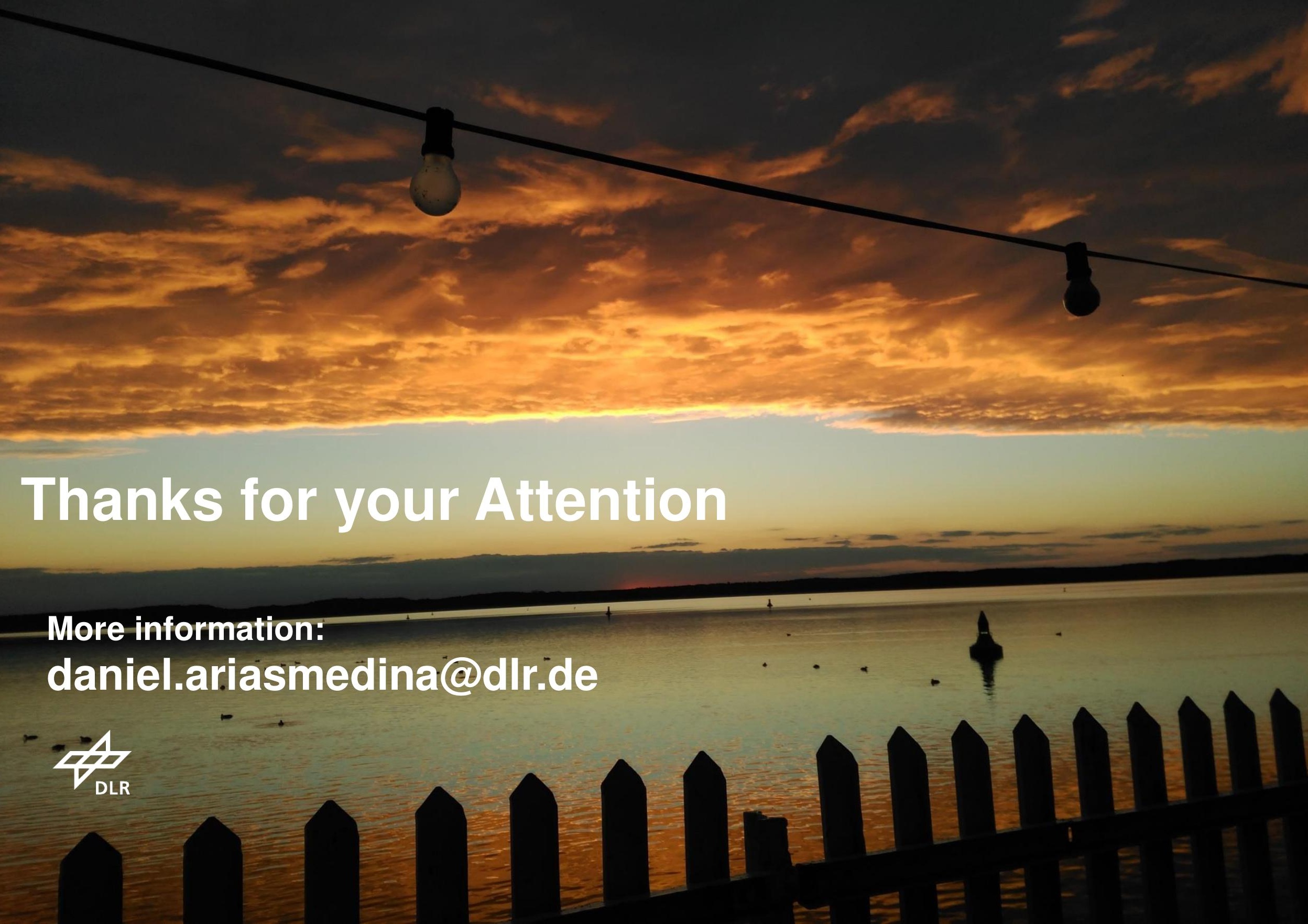